Conclusions This hospice model has demonstrated significant reductions in hospital admissions and increased confidence of care home staff in end of life practices. It has the potential to be expanded in other localities.

\section{0-20 WHEN 999 GETS CALLED AT THE END OF LIFE: EQUIPPING AMBULANCE CLINICIANS TO PROMOTE A DIGNIFIED DEATH (HOSPICES AND AMBULANCE SERVICE WORKING TOGETHER)}

${ }^{1}$ Claire Nicell, ${ }^{2}$ Richard Smith, ${ }^{3}$ James Hill, ${ }^{4}$ Sarah Stead. ${ }^{1}$ The Hospice of St Francis, Berkhamsted, UK; ${ }^{2}$ East of England Ambulance Service NHS Trust, Watford, UK; ${ }^{3}$ East of England Ambulance Service NHS Trust, Chelmsford, UK; ${ }^{4}$ East of England Ambulance Service NHS Trust, Bedford, UK

\subsection{6/bmispcare-2017-hospice.20}

Background End of life care is an emergent aspect of ambulance clinicians' work. They are often called to an emergency or crisis in an individual's last days of life and have to make complex decisions with limited information. However they receive limited formal palliative care education. Two hospices were approached by a local paramedic to see if they could help address this educational need.

Aim of the Collaborative Work

- For ambulance clinicians to gain confidence in recognising end of life needs of patients and their families and delivering appropriate care.

- For hospice professionals to understand the role and abilities of ambulance clinicians in delivering end of life care.

What have we achieved?

- Two hospices and their local Ambulance NHS Trust worked collaboratively to devise a one-day workshop for paramedics to explore palliative and end of life care.

- The workshop included communication skills, symptom control, recognising dying, promoting patient wishes and accessing local palliative care services.

- Feedback from 70+clinicians was good and their confidence scores increased

- 'Helps make a grey area of our work more understood.'

- Two paramedics completed the European Certificate in Essential Palliative Care and are recognised locally as palliative care champions within their work in the community and control room.

- Links established with local university; end of life care sessions delivered to third-year paramedic students and palliative care student placements arranged.

Additional Benefits

- Increased mutual understanding of practitioners' roles informing practice and education.

- Paramedics accessing ongoing communication skills training.

- Paramedics delivering teaching to hospice staff highlighting end of life care challenges.

- Improved communication between ambulance crews and local hospices.

- Joint hospice and ambulance end of life care Facebook discussion delivered as part of the Ambulance's service End of Life Care Awareness month

- Joint end of life care strategy working.

\section{Lessons learnt in engagement and governance}

\section{0-21 DRIVING CULTURE CHANGE: CROSS-ORGANISATIONAL ENGAGEMENT WITH PEOPLE WITH PERSONAL EXPERIENCE}

Andrea Dechamps. St Wilfrid's Hospice, Eastbourne, UK

10.1136/bmjspcare-2017-hospice.21

Background User involvement in hospices, whilst acknowledged as important, can be viewed as particularly difficult with people nearing end of life, can become the sole domain of a particular worker/expert (e.g., the palliative care social worker) and can lack overall buy-in from across organisations. Two years ago our hospice disbanded our main vehicle for user involvement, the Patient Forum, in their own judgement by now weary and unoriginal. Under a PPE (People with Personal Experience) governance stream we embarked on an open-ended, ambitious drive innovating engagement with PPE. Aims

- To work towards cross-organisation engagement with PPE, through multiple time-limited PPE initiatives led by volunteers, clinical and non-clinical staff at all levels and across settings.

- To instil a sense of curiosity and develop competence amongst all staff and volunteers regarding PPE involvement.

- Ultimately, for PPE involvement to significantly influence service delivery and development across settings.

Methods

- Definition of PPE revised to include our patients, families, customers, public, community, staff and volunteers.

- Key performance target set at 20 PPE initiatives for 2016/17 demonstrated senior commitment.

- Support given to project leaders, with focus on '(almost) anything goes'

- Initiatives mapped by project leaders against business priorities, CQC Key Lines of Enquiry, Quality Improvement Priorities and themes from complaints.

Results Twenty diverse initiatives delivered 2016/2017. Two showcasing events. Demonstrable changes to service delivery across the organisation resulting from above plus culture change amongst staff and volunteers.

'I am just beginning to understand what PPE involvement means - it matters!'

'Involving families in how we design $\mathrm{xx}$, designing $\mathrm{xx}$ in collaboration, has made a real difference.'

Conclusion The above describes the first significant stage of a new approach to PPE involvement. We already have demonstrable evidence of quality improvement as a result plus culture change. We believe the described approach to be innovative and have potential for duplication in other settings.

\section{0-22 CQC REQUIRES IMPROVEMENT TO OUTSTANDING IN ONE YEAR: A HOSPICE JOURNEY}

Kerry Harrison. Heart of Kent Hospice, Aylesford, UK

10.1136/bmjspcare-2017-hospice.22 
Background Inspected in February 2016 the hospice achieved an outstanding rating for Caring and requires improvement for four other key lines of enquiry, Safe, Effective, Responsive and Well-Led, including three breaches of legislation. A new leadership team had been in place for a short while and had already identified an action plan and programme for improvements.

Aims There was a lack of audit, quality assurance and governance within the organisation. Reporting of incidents was underpinned by a blame culture which acted as a barrier to incidents being reported, leading to a lack of learning and accountability. Longevity of service amongst some clinical staff created an environment that was resistant to change and a lack of recognising the need to improve and monitor practice. Method A priority was to recruit to key posts including a new Registered Manager and Head of Information and Quality, a team who had the knowledge, skills and ability to engage clinical teams in the change process. Education sessions were carried out to explore how to use incidents as learning experiences to implement changes to practice and improve accountability. A new competency framework was introduced. An audit and research group was set up to facilitate audit becoming routine practice. Quality and data spot checks were carried out monthly. A clinical management team regularly reviewed policies and guidelines in line with legislation and national standards. Improvements were underpinned by improving the patient/carer experience and outcomes: Advance Care Plans, Patient Centred Goals, and Preferred Place of Death. There was investment in workforce wellbeing: clinical supervision, Schwartz Rounds and a representative group.

Outcome Inspection in February 2017 achieved 'Outstanding' in Caring and Responsive, 'good' in Safe, Effective and WellLed. Overall achieved outstanding, and a delighted workforce. The inspectors noted 'impressive improvements and a learning culture throughout the hospice'.

\section{O-23 FROM CRISIS TO OPPORTUNITY}

Gill Horne, Rachael Bryett, Jon Hill, Georgina Walker. Rowcroft Hospice, Torquay, UK

\subsection{6/bmjspcare-2017-hospice.23}

Background Rowcroft Hospice delivered community, inpatient, hospice@home and chronic oedema services to a population of 280000 over 300 sqm. By Christmas 2015 it was clear that legacy income was going to fall well short of the budget; a risk we carried for many years was now realised. Our plans had proved unsustainable and the trustees had to make the decision whether to close the hospice or significantly reduce services.

Aims To reduce the hospice's financial deficit and bring financial stability, including replenishing sufficient reserves for Board assurance, whilst retaining 'core' patient and family services and our reputation for outstanding care.

\section{Methods}

1. Develop plans to redesign and reduce patient, family and support services to those deemed 'core' within four months.

2. Launch a Crisis appeal for $£ 585000$
3. Establish 'Project Red' including staff and volunteer briefings, a collective consultation, involvement of trade unions, staff empowerment to negotiate savings within teams

4. Develop a transformation plan

Results Plans were developed through collaboration with the local NHS provider and CCG. Our chronic oedema service was taken over by the NHS. We reduced from 17 to 12 inpatient beds - redesigning a bay into a dayroom; reduced staffing of hospice@home and redesigned our community and support services. We have delivered similar service activity to the previous year, although our contacts are fewer. We exceeded our crisis appeal target by $£ 20000$ and gained 500 new regular givers. Our transformation plan has meant closer engagement with our local NHS provider and other partners. Conclusion Although born out of crisis, the opportunities for learning, redesigning services to fit within a reduced budget, supporting team cohesiveness and a 'one hospice' culture, enhancing our collaborations with the local NHS provider and CCG and in gaining support from the public and partners have all served to secure the hospice's position.

\section{Parallel session 6: Leaning in: transformation and privilege}

\section{0-24 VIRTUAL REALITY TRANSFORMING THE LIVES OF TERMINALLY ILL PATIENTS}

Katherine Pabla, John Knight, Mandy Motley. LOROS, Leicester, UK

\subsection{6/bmjspcare-2017-hospice.24}

We have commissioned and produced a virtual reality film of a local park to give terminally ill patients whose lives have become restricted due to their illness, the chance to see the world from the comfort of their chair or bed. By simply wearing the virtual reality headset, patients are 'virtually transported' to a completely different location, and are then able to discuss and reminisce with friends and families. With patients' wellbeing in mind, the films are an important therapeutic tool, relaxing those that watch as they are 'taken away' to a familiar environment. Research suggests that the brain accepts the virtual world within $20 \mathrm{~s}$ after which the experience becomes all absorbing. A patient who has Motor Neurone Disease (MND), was the first patient to try out the experience. 'You soon relax, it's just like you're there, I loved it' he said, as he experienced 'walking through' the park. 'I nearly waved at somebody, as they walked past.' 'Since being diagnosed with MND, we can get out but I can't spend a lot of time out of the wheelchair, so being able to have these experiences through the glasses is really good'. 'It's almost as good as the real thing.' We are now looking at enhancing patients' experiences both regionally and nationally, by partnering with other hospices to commission a portfolio of films, like walking on a beach, or a canal ride for example. These films will be accessible via a bespoke app, allowing multiple patients to access the films at the same time. The app will also allow shared experiences for groups such as in day 